\title{
Muon Cooling: Longitudinal Compression
}

\author{
Yu Bao, ${ }^{1}$ Aldo Antognini, ${ }^{2,}$ Wilhelm Bertl, ${ }^{1}$ Malte Hildebrandt, ${ }^{1}$ Kim Siang Khaw, ${ }^{2}$ Klaus Kirch, ${ }^{1,2}$ Angela Papa, \\ Claude Petitjean, ${ }^{1}$ Florian M. Piegsa, ${ }^{2}$ Stefan Ritt, ${ }^{1}$ Kamil Sedlak, ${ }^{1}$ Alexey Stoykov, ${ }^{1}$ and David Taqqu ${ }^{2}$ \\ ${ }^{1}$ Paul Scherrer Institute, 5232 Villigen-PSI, Switzerland \\ ${ }^{2}$ Institute for Particle Physics, ETH Zurich, 8093 Zurich, Switzerland
}

(Received 11 February 2014; published 4 June 2014)

\begin{abstract}
A $10 \mathrm{MeV} / c$ positive muon beam was stopped in helium gas of a few mbar in a magnetic field of $5 \mathrm{~T}$. The muon "swarm" has been efficiently compressed from a length of $16 \mathrm{~cm}$ down to a few mm along the magnetic field axis (longitudinal compression) using electrostatic fields. The simulation reproduces the low energy interactions of slow muons in helium gas. Phase space compression occurs on the order of microseconds, compatible with the muon lifetime of $2 \mu \mathrm{s}$. This paves the way for the preparation of a highquality low-energy muon beam, with an increase in phase space density relative to a standard surface muon beam of $10^{7}$. The achievable phase space compression by using only the longitudinal stage presented here is of the order of $10^{4}$.
\end{abstract}

DOI: 10.1103/PhysRevLett.112.224801

PACS numbers: 29.27.-a, 14.60.Ef, 29.38.Db, 82.20.Tr

Standard muon $\left(\mu^{+}\right)$beams have a relatively high energy and poor phase space quality. A new scheme has been proposed making use of stopped $\mu^{+}$in a He gas target [1]. Through the stopping process high intrinsic phase space compression is achieved. The remaining challenge is to extract the muons fast enough into vacuum. This is done by compressing the stopped muon swarm with electric fields and guiding it into a small extraction hole. In this Letter we report the successful demonstration of muon swarm compression along the magnetic field lines.

Related schemes have been used in the field of rare isotope investigations [2,3]. High energy ion beams are stopped in He gas and compressed. Typical manipulation and extraction times are 5-200 ms. For muons, much faster techniques are vital.

The new concept for fast compression is based on a position-dependent muon drift velocity $\vec{v}_{D}$ in gas. In a long He gas target placed in a longitudinal high magnetic field, the stopping muons are first transversely, then longitudinally compressed and finally extracted through a $1 \mathrm{~mm}^{2}$ side hole in the transverse direction (see Fig. 1). The operation takes place in $8 \mu \mathrm{s}$ [1].

This tertiary beam line is an add on to a secondary surface muon beam line, reducing the phase space of the input beam by $10^{10}$ with $10^{-3}$ efficiency.

By using, e.g., the PSI $\mu \mathrm{E} 4$ surface beam with $4 \times 10^{8} \mu^{+} / \mathrm{s}$, an eV-energy beam can be obtained with $1 \mathrm{~mm}^{2}$ transverse area and intensity of $5 \times 10^{5} \mu^{+} / \mathrm{s}, 2$ orders of magnitude larger than presently achieved [4]. This development is also highly attractive in view of the HiMB project [5] aiming at a surface muon beam of $\sim 10^{10} \mu^{+} / \mathrm{s}$. Space charge effects will limit the output intensity of the compressed beam to about $1 \times 10^{7} \mu^{+} / \mathrm{s}$.

It is important to note that depolarization during muon swarm compression is only a few percent because of the strong longitudinal magnetic field [6]. Thus, starting from highly polarized muons, such a beam can be used for next generation $\mu \mathrm{SR}$ applications, muonium (Mu) spectroscopy, a muon $g$-2 experiment, searches for a muon electric dipole moment, and $\mathrm{Mu}-\overline{\mathrm{Mu}}$ conversion. Because of space charge effects and muon losses it is not suited for muon collider projects.

The drift velocity of charged particles in gas in the presence of electric $\vec{E}$ and magnetic $\vec{B}$ fields is [7]

$$
\vec{v}_{D}=\frac{\mu E}{1+\omega^{2} \tau_{c}^{2}}\left[\hat{E}+\omega \tau_{c} \hat{E} \times \hat{B}+\omega^{2} \tau_{c}^{2}(\hat{E} \cdot \hat{B}) \hat{B}\right],
$$

where $\hat{E}$ and $\hat{B}$ are the unit vectors along $\vec{E}$ and $\vec{B}, \omega=$ $e B / m$ the cyclotron frequency with $m$ the muon mass, $\mu$ the muon mobility in the gas, and $\tau_{c}$ the mean time between

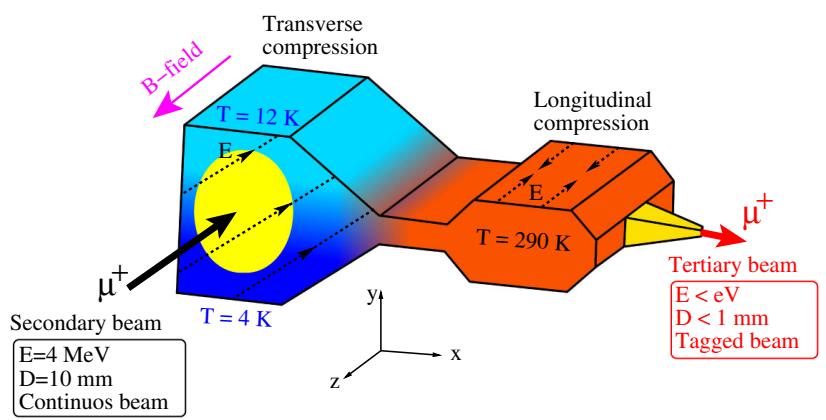

FIG. 1 (color online). Schematic of the beam line proposed in [1]. $\mu^{+}$traveling in the $-z$ direction are stopped in 5 mbar He gas at cryogenic temperatures. First, transverse ( $y$ direction) compression occurs within a density gradient in He gas (blue region). Then at room temperature the longitudinal ( $z$ direction) compression takes place (red region). In the yellow region a mixed transverse-longitudinal compression precedes before extraction into vacuum. 
collisions. For small $\omega \tau_{c}$ the muon drift is along the electric field lines. This is the regime of high gas density where the collision rate is large. For large $\omega \tau_{c}$ the third term dominates and muons follow the magnetic lines.

The beam line we are developing is composed of a sequence of stages as shown in Fig. 1. In the initial transverse compression stage a $\mu^{+}$beam is stopped in $\mathrm{He}$ gas at 5 mbar pressure inside a $5 \mathrm{~T}$ longitudinal $B$ field $\hat{B}=(0,0,1)$ and a transverse electric field $\hat{E}=1 / \sqrt{2}(1,1,0)$. The gas temperature in this first stage has a vertical gradient from 4 to $12 \mathrm{~K}$. At lower densities (top part) $\tau_{c}$ is large and $\vec{v}_{D}$ is dominated by the $\hat{E} \times \hat{B}$ term. Hence, the muons drift diagonally in the $-y$ direction and in the $+x$ direction. By contrast, at larger densities (bottom part) the first term of Eq. (1) dominates and the resulting drift velocity is along $\hat{E}$. Therefore, muons originating from the upper (lower) part of the stop distribution while drifting in the $+x$ direction are moving downwards (upwards) giving rise to a compression in the transverse $(y)$ direction. The $10 \mathrm{~mm}$ wide stopping volume in the $y$ direction is thereby reduced to a swarm of muons moving in the $x$ direction with a height of $0.5 \mathrm{~mm}$ (in the $y$ direction) and a length (in the $z$ direction) of $50 \mathrm{~cm}$ or more [1].

The muon swarm is then entering a second stage at room temperature (low density) where longitudinal compression (in the direction of $\hat{B}$ or $z$ ) takes place. Hence, the muons are directed towards the exit hole as shown in Fig. 1. The third term of Eq. (1) is here dominant. The electric fields are designed such that $E_{z}$ changes sign in the center of this region. Thus, muons drift to the center along the magnetic field lines, which gives rise to longitudinal compression. A nonvanishing $E_{y}$ component guarantees that the muon swarm drifts also along the $+x$ axis because of the $\hat{E} \times \hat{B}$ term. An additional muon swarm compression in both the $y$ and $z$ direction occurs in a third stage followed by vacuum extraction through a small orifice.

In this Letter we present an experimental demonstration of compression along the magnetic field lines, i.e., longitudinal compression. A standard $\mu^{+}$beam is stopped in a few mbar $\mathrm{He}$ gas at room temperature and $5 \mathrm{~T}$ longitudinal magnetic field. The elongated stopping distribution is compressed into the minimum of a $V$-shaped electrostatic potential as shown in Fig. 2. From the time distribution of the muon-decay positrons $\left(e^{+}\right)$detected with two positron counters $\left(P_{1}, P_{2}\right)$ placed in the vicinity of this potential minimum it is possible to quantify the muon drift.

The experiment was performed at the PSI $\pi E 1$ beam line tuned to deliver $\mu^{+}$of $10 \mathrm{MeV} / c$ momentum $(500 \mathrm{keV}$ energy). After elimination of the positron contamination with a electromagnetic separator, the $\mu^{+}$beam is focused into a $5 \mathrm{~T}$ solenoid containing a $\mathrm{He}$ gas target at a few mbar pressure. A $\mu^{+}$rate of $2 \times 10^{4} \mathrm{~s}^{-1}$ was measured using a $30 \mu \mathrm{m}$ thick plastic scintillator $\left(S_{1}\right)$ of $10 \mathrm{~mm}$ diameter read out by four Geiger-mode avalanche photodiodes. $S_{1}$ provides the event start time.

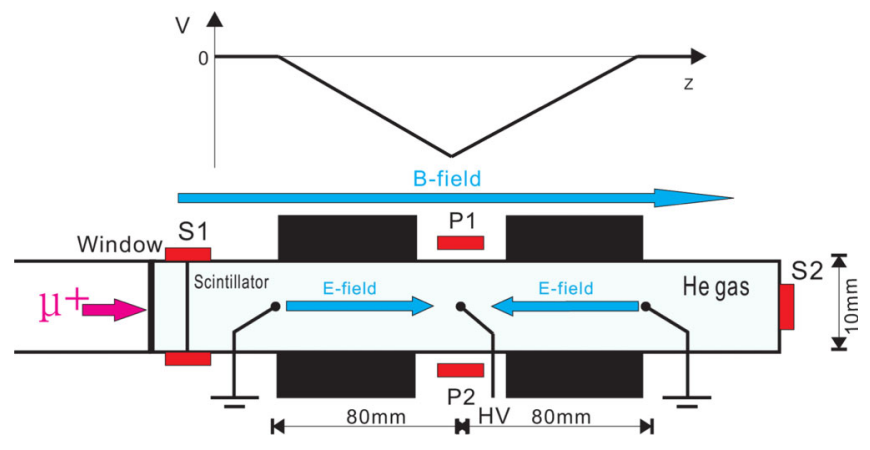

FIG. 2 (color online). Setup in the $5 \mathrm{~T}$ solenoid. Muons enter the gas target, cross the entrance detector $S_{1}$, and a fraction stops in the He gas. The stopped $\mu^{+}$are compressed along the $B$ field using a $V$-shaped electric potential. $P_{1}$ and $P_{2}$ are $e^{+}$counters. $S_{2}$ is a scintillator counter for aligning the muon beam.

The beam momentum and the scintillator thickness were chosen to give the highest (few \%) fraction of $\mu^{+}$stops in the $160 \mathrm{~mm}$ long "active" region of the few mbar He target. The majority of the $\mu^{+}$passes through the gas and reaches a second detector $S_{2}$ which is also used to align the target along the $B$-field lines.

The inner walls of the target are made of printed circuit boards (PCB) covered by thin metallic strips used to define the $V$-shaped electrostatic potential. To compress the $\mu^{+}$to the center $(z=0)$ of the target a negative HV is applied to the central strips. The other strips are at decreasing absolute potential till $z= \pm 80 \mathrm{~mm}$, where ground potential is applied.

The $S_{1}, S_{2}, P_{1}$, and $P_{2}$ signals are recorded with a DRS4 [8] waveform digitizer triggered by $P_{1}$ or $P_{2}$. Figure 3 shows $e^{+}$time distributions measured in $P_{1}$ and $P_{2}$ relative to $S_{1}$. To remove the effect related to the $\mu^{+}$decay, the histograms have been scaled with $\exp (t / \tau)$, where $t$ is the time and $\tau=2.2 \mu \mathrm{s}$ the $\mu^{+}$lifetime. The "prompt" peaks originate from the higher energy $\mu^{+}$decaying in flight in the acceptance region (between $\pm 18 \mathrm{~mm}$ in the $z$ direction) of $P_{1}$ and $P_{2}$.

When a negative $\mathrm{HV}$ is applied, the $\mu^{+}$are attracted close to $P_{1}$ and $P_{2}$. The resulting $e^{+}$time distributions (blue, + ) show an increase caused by the muon drift into a region with higher positron detection acceptance. On the contrary, applying a positive $\mathrm{HV}$, the $\mu^{+}$are pushed out of the acceptance region resulting in a decrease of detected positrons (green empty circles).

The curve for large positive $\mathrm{HV}$ at delayed time is equivalent to a background measurement. Its flat asymptotic behavior reveals that this background is muon-correlated. It arises from $\mu^{+}$stopping in the target walls, in the beam collimators, in the beam dump, and in the inactive region of the target.

A relevant difference between the conditions of this experiment and those in the longitudinal compression stage of [1], is that in [1] the $\mu^{+}$enter the longitudinal 


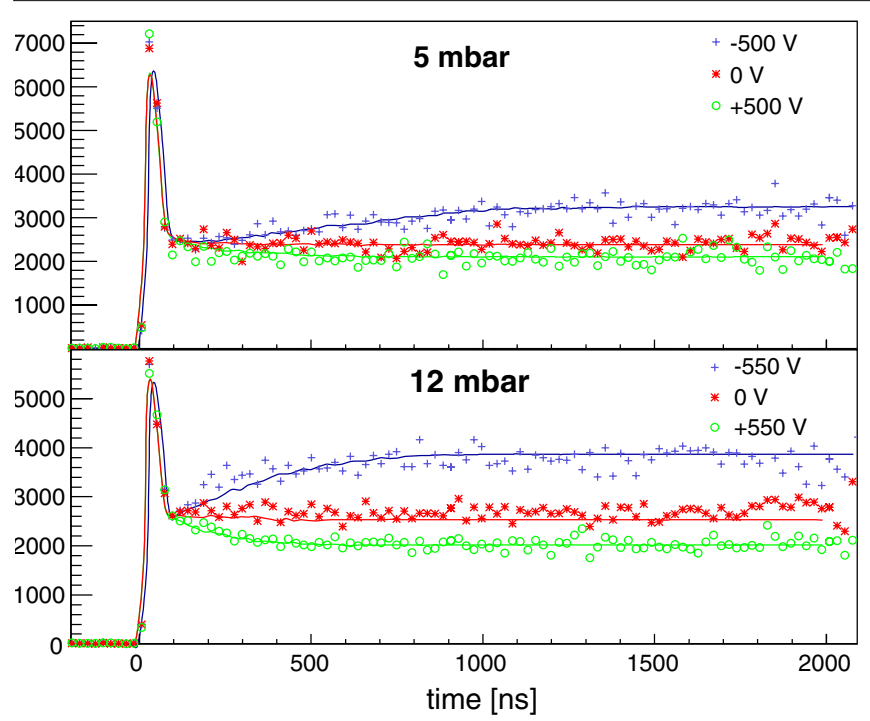

FIG. 3 (color online). Measured and simulated (continuous lines) positron time spectra multiplied with $e^{t / 2200}$ for two different pressures, where $t$ is the time in ns. The simulations account for muon chemical capture with a rate of $40 \times 10^{6} \mathrm{~s}^{-1}$ and a misalignment between the target and $B$ field of $1 \mathrm{mrad}$ (see text).

compression stage at an energy around $1 \mathrm{eV}$, while in the experiment presented here, the muons enter the active volume at $\mathrm{keV}$ energies. Thus, here the slowing-down process competes and is mixed with the drift induced by the electric field.

To analyze the measured time spectra (which include prompt peak, background, slowing down, and compression) and quantify the compression, we extended the GEANT4 simulation package [9] to include $\mu^{+}$physics in the $0.1 \mathrm{eV}-1 \mathrm{keV}$ energy range. Below $1 \mathrm{keV}$ energy standard GEANT4 processes have been switched off and elastic $\mu^{+}-\mathrm{He}$ collision, $\mathrm{Mu}$ formation and $\mathrm{Mu}$ ionization have been implemented. Among these processes the elastic $\mu^{+}-\mathrm{He}$ scattering is the decisive interaction that controls the muon compression. It has been included in the Monte Carlo (MC) simulation starting from the proton differential cross sections calculated in [10] adapted to muons using energy scaling [11].

When a $\mu^{+}$crosses matter in the $\mathrm{keV}$ energy regime it undergoes charge exchange, that is electron-capture and electron-loss processes. Both processes have been accounted for separately in our MC simulation using the velocity scaled cross sections of [12]. The stopping power caused by these processes is given in Fig. 4, where for simplicity only the net $\mu^{+}$energy losses caused by the full charge exchange cycle is represented without separating electron-capture and electron loss. The $\mu^{+}$stopping power, indicated by the circles in Fig. 4, which results from velocity scaling the measured proton stopping power [13], deviates from the stopping power given by the charge exchange because it also includes non-negligible processes

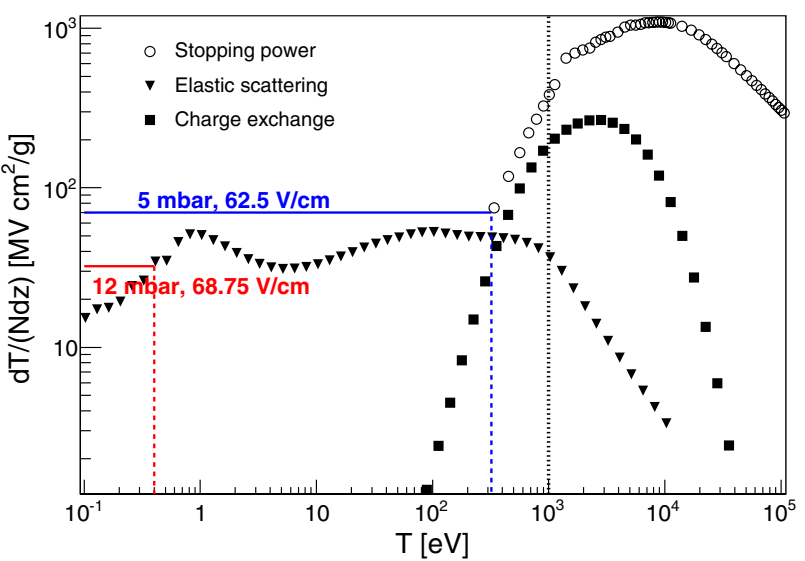

FIG. 4 (color online). Energy loss processes of $\mu^{+}$at low energy scaled from proton data. The empty circles show the stopping power caused by $\mathrm{He}$ ionization, $\mathrm{He}$ excitation, and charge exchange processes. The curve with squares accounts for energy losses due to $\mathrm{Mu}$ formation and ionization. The curve with triangles represents the kinetic energy loss $[d T /(N d z)]$ caused by elastic scattering per unit length in a fixed direction $(z$, electric field direction). The vertical dashed lines (blue, red) are the resulting equilibrium energies at two experimental conditions.

such as ionization and excitation of the target helium atom. Because no cross sections are available for these processes, between $300 \mathrm{eV}$ and $1 \mathrm{keV}$ we have adapted the energy losses of the charge exchange processes to reproduce the stopping power scaled from proton data. This approximation is good enough since simulations show that the drift of the muon is insensitive to the precise implementation of these processes, as muons spend only a small fraction of time in this energy range.

The curve shown with triangles in Fig. 4 illustrates the average kinetic energy $(T)$ loss of diffusive motion per unit length along a given direction $(d z)$ when only elastic scattering takes place. This curve has been obtained from the diffusion theory of [14] using the energy scaled transport cross sections of [10]. The horizontal red and blue lines represent the kinetic energy gain per unit displacement in the $z$ direction due to an electric field (in the $z$ direction). By equating energy loss caused by collisions, with energy gain due to the applied electric field, one can estimate the average muon equilibrium drift energy at the corresponding field and density conditions. For example, as shown in Fig. 4 at $E=68.75 \mathrm{~V} / \mathrm{cm}$ and a pressure of $12 \mathrm{mbar}$, the average kinetic energy is $0.4 \mathrm{eV}$. As $E / N$ increases $(N$ being the gas number density), the average equilibrium energy increases. If $E / N$ is larger than the local maximum at around $1 \mathrm{eV}$ energy, muons are accelerated to much higher kinetic energies as shown by the vertical (blue, dashed) line. At this point, the energy gain in the $E$ field is always larger than the energy loss due to elastic collisions. Hence, muons are continuously accelerated ("run-away") until other processes (charge exchange and inelastic collisions) limit their speed $[14,15]$. Fast longitudinal 


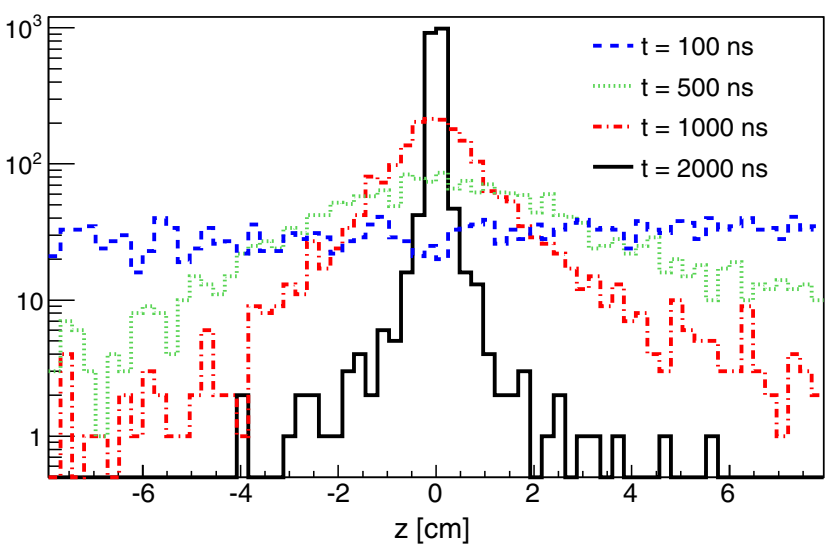

FIG. 5 (color online). Muon distribution at various times during the compression for 5 mbar and $\mathrm{HV}=-550 \mathrm{~V}$.

compression of the $\mu^{+}$swarm within 10-15 cm length requires the $\mu^{+}$to be accelerated at run-away conditions. The equilibrium energies computed with our MC simulations based on differential cross sections reproduce the equilibrium energies obtained from the diffusion theory in [14] and shown in Fig. 4.

A simulated time evolution of the muon spatial distribution during compression in the target is given in Fig. 5 . Starting from an approximately flat stop distribution $(t=100 \mathrm{~ns})$, the muons are compressed in the center in less than $2 \mu \mathrm{s}$.

The positron time spectra of $P_{1}$ and $P_{2}$ simulated with our MC program are shown in Fig. 6. The 12 mbar with $\mathrm{HV}=-550 \mathrm{~V}$ (continuous) curve has a fast and a slow component. The slow component which becomes visible at times later than $700 \mathrm{~ns}$ arises from slow muons drifting at the equilibrium energy of $0.8 \mathrm{eV}$. The fast component results from compressing muons while having several

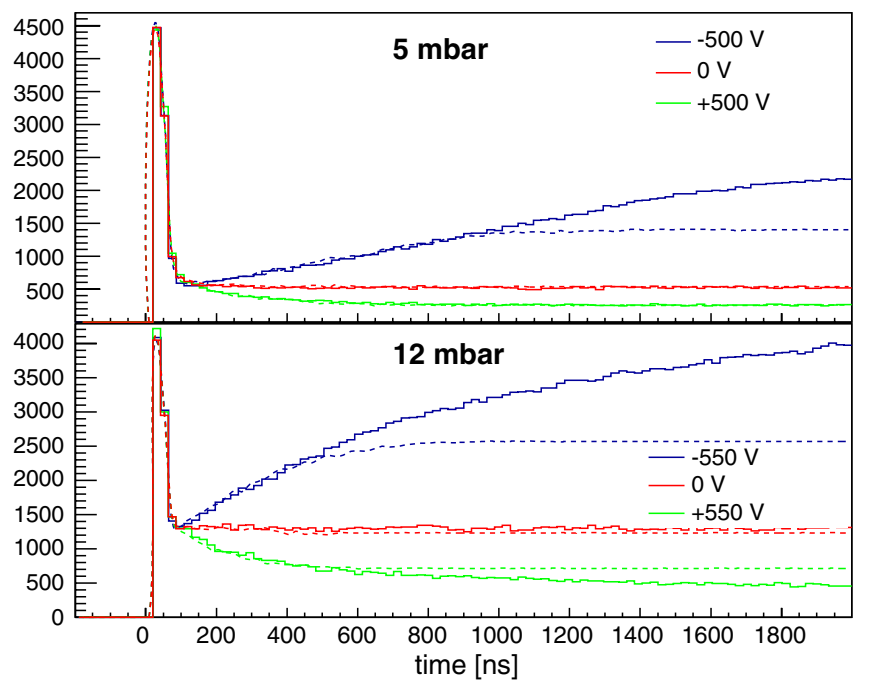

FIG. 6 (color online). Simulated $e^{+}$time spectra without (continuous lines) and with (dashed lines) muon chemical capture to impurities with $R=40 \times 10^{6} \mathrm{~s}^{-1}$ and $10 \mathrm{eV}$ cutoff. hundreds eV energy, thus, still in the process of slowing down to the equilibrium energy. For 5 mbar no fast and slow component can be distinguished since the equilibrium drift energy is higher (around $200 \mathrm{eV}$ ) and the muon deceleration process from the $100 \mathrm{keV}$ energy regime is slower. Hence, most muons reach the center of the target before they slow down.

The measured time spectra of Fig. 3 do not show any hint of a slow component. At 12 mbar the measured compression is completed at about $500 \mathrm{~ns}$. According to simulations, at this time the $\mu^{+}$energy ranges from 1 to $10 \mathrm{eV}$. The probable explanation for the absence of the slow component is that at these energies the muon interacts with impurities to form muonic ions or to replace a proton of these molecules ("chemical capture"). We therefore introduced into the MC simulations a constant loss rate $R$ in the low energy range up to a cutoff energy which was taken to be $10 \mathrm{eV}$. Large desorption rates have been measured in our target (PCB glued with araldite) giving rise to impurities of about $10^{14}$ molecules $/ \mathrm{cm}^{3}$ corresponding to few atomic monolayers when integrated over the active target length. It is known that few monolayers of impurities on the Armoderator surface of the LEM beam line [16] are sufficient to reduce by 1 order of magnitude the number of $\mu^{+}$with energy between 0 to $30 \mathrm{eV}$ emerging from the surface [17]. The dotted curves in Fig. 6 show the effect of this chemical capture assuming a rate of $R=40 \times 10^{6} \mathrm{~s}^{-1}$ for energies $<10 \mathrm{eV}$. These values where chosen to fit the measured data.

The measured time distributions show a slightly larger flat (muon correlated) background than the simulations. This can be explained by a small (about $1 \mathrm{mrad}$ and within uncertainties) misalignment between the magnetic field lines and the target, causing an increased number of $\mu^{+}$ stopping in the target walls in front of the positron detectors. Adjusting the simulations with such misalignment and including chemical capture, yields complete agreement with the measured spectra as shown in Fig. 3. Variation of the elastic $\mu^{+}-\mathrm{He}$ cross section by a factor of 2 leads to simulated time spectra which disagree strongly with the measurements.

In conclusion, we have demonstrated the compression of stopping muons along the magnetic field direction. This demonstration relies on the agreement between the experimental results and simulations based on GEANT4 extended to account for low energy processes. For the first time run-away conditions for muons [14] have been experimentally exploited. Applying our MC program to muons starting the process at low energy as in [1], yields a longitudinal compression process with almost no losses in walls or muonium formation in less than $2 \mu \mathrm{s}$.

Using an additional electric field, these muons can be drifted $(E \times B$ term) in the $x$ direction and extracted into vacuum through an orifice of $\sim \mathrm{mm}^{2}$ size. Their average energy would be a few $\mathrm{eV}$ before being reaccelerated by a 
pulsed electric field to $\mathrm{keV}$ energies. Including lifetime losses, stopping and extraction efficiencies, the initial phase space density is increased by a factor of $10^{4}$ due to the slowing down and longitudinal compression.

We are grateful to machine and support groups of the Paul Scherrer Institute and of ETH Zurich. We acknowledge the help of F. Kottmann, R. Scheuermann, T. Prokscha, D. Reggiani, K. Deiters, T. Rauber, O. Kiselev, Y. Lee, M. Diepold, S. Beuret, Z. Hochmann, and F. Barchetti. The work was supported by the SNF Grant No. 200020_146902.

* Corresponding author. aldo@phys.ethz.ch

[1] D. Taqqu, Phys. Rev. Lett. 97, 194801 (2006).

[2] G. Bollen et al., Nucl. Instrum. Methods Phys. Res., Sect. B 266, 4442 (2008).

[3] G. Savard, Eur. Phys. J. A 25, 713 (2005).

[4] T. Prokscha, E. Morenzoni, K. Deiters, F. Foroughi, D. George, R. Kobler, A. Suter, and V. Vrankovic,
Nucl. Instrum. Methods Phys. Res., Sect. A 595, 317 (2008).

[5] A. Blondel et al., arXiv:1301.6113.

[6] M. Senba, J. Phys. B 31, 5233 (1998).

[7] Instrumentation in High Energy Physics, edited by F. Sauli (World Scientific, Singapore, 1992).

[8] S. Ritt, http://www.psi.ch/drs/.

[9] S. Agostinelli et al., Nucl. Instrum. Methods Phys. Res., Sect. A 506, 250 (2003).

[10] P. S. Krstic and D. R. Schultz, At. Plasma-Mater. Interact. Data Fusion 8, 337 (1998).

[11] M. Senba, J. Phys. B 22, 2027 (1989).

[12] Y. Nakai, T. Shirai, T. Tabata, and R. Ito, At. Data Nucl. Data Tables 37, 69 (1987).

[13] J. F. Ziegler, SRIM: The Stopping and Range of Ions in Matter (2011), http://srim.org.

[14] E. A. Mason, S. L. Lin, and I. R. Gatland, J. Phys. B 12, 4179 (1979).

[15] F. Howorka, F. C. Fehsenfeld, and D. L. Albritton, J. Phys. B 12, 4189 (1979).

[16] T. Prokscha, E. Morenzoni, K. Deiters, F. Foroughi, D. George, R. Kobler, A. Suter, and V. Vrankovic, Nucl. Instrum. Methods Phys. Res., Sect. A 595, 317 (2008).

[17] E. Morenzoni et al., J. Appl. Phys. 81, 3340 (1997). 